\title{
From Spider Webs to a Biomimetic Optical Fibre Sensor
}

\author{
Kenny Hey Tow ${ }^{1}$, Desmond M. Chow ${ }^{1}$, Fritz Vollrath ${ }^{2}$, Isabelle Dicaire ${ }^{3,5}$, Tom \\ Gheysens $^{4,5}$ and Luc Thévenaz \\ ${ }^{1}$ EPFL Swiss Federal Institute of Technology, Group for Fibre Optics, SCI-STI-LT, Station 11, CH-1015 Lausanne, \\ Switzerland \\ ${ }^{2}$ University of Oxford, Department of Zoology, Oxford OX1 3PS, UK \\ ${ }^{3}$ CCTT Optech, 1111 Lapierre St., Montreal QC, H8N 2J4, Canada \\ ${ }^{4}$ Polymer Chemistry \& Biomaterials Group, Ghent University, Krijgslaan 281, S4-Bis, B-9000 Ghent, Belgium \\ ${ }^{5}$ Advanced Concepts Team, European Space Agency (ESA), Noordwijk, The Netherlands. \\ kenny.heytow@epfl.ch
}

\begin{abstract}
Can we use spider silk threads as natural, biological optical fibre sensors? In this communication, we will see how we can harness the optical properties of spider dragline silk and use it for sensing. (C) 2018 The Author(s)
\end{abstract}

OCIS codes: (060.2370) Fiber optics sensors; (060.2400) Fiber properties; (060.2300) Fiber measurements

\section{Introduction}

Biological receptors work at the nanoscale level with minimal energy, occupying very small spaces, high sensor density, accuracy and robustness. Olfactory receptors, responsible for detecting odorants that give the sense of smell, display affinity for a range of odour molecules. Upon binding to a single odorant molecule, they undergo structural conformation changes, which trigger an action potential in the receptor neuron via a second messenger pathway sending a signal to the nervous system, which then identifies the smell [1].

A similar biomimetic approach, analogous to the sense of smell, can be adopted in the field of optical fibre sensing to produce less complex and more efficient fibre-optic chemical sensors (FOCS). Owing to their 'cheap and cheerful' reputation, conventional silica optical fibres are still extensively used for detecting chemical species. Indeed, one can easily find in the literature an exhaustive list of FOCS, which are silica optical fibre-based devices used to measure gases, vapours (hydrocarbons, organic solvents, etc.) and inorganic ions (e.g. to determine the $\mathrm{pH}$ level) even though, by essence, silica is notoriously known to be a chemically inert material. In fact, the silica optical fibre is reduced to, merely, the role of a conduit to bring light to and from a chemically-sensitive coating in these sys-

tems and is not exploited as the sensing element in FOCS. Their sensing mechanism is either indirect - the reaction between the analyte and a doped fibre-tip end modifies the environmental quantities for which silica is sensitive - or part of the light must be made to propagate out of the silica fibre, for instance by evanescent field, and interact with a chemically sensitive coating placed on the fibre. In the first case, the light interaction is confined to a restrictive sensing area (fibre tip) while for the latter the waveguiding properties of the silica fibre is clearly a penalty.

The efficiency of these systems can be disruptively increased by using natural protein optical fibres, capable of interacting with the surrounding environment in their pristine condition instead of their silica counterpart. By analysing the change in material properties of structural receptor-proteins upon interacting molecules, specific compounds inducing characteristic conformational changes resulting in distinct material property changes can be identified.

For instance, hydrophilic materials such as cellulose fibrils in wheat awns, horse hair, wool, etc. all swell during bulk water uptake. These specific conformational changes lead to a signature change in material properties of the fibre 
bulk material, which can be measured by monitoring the parameters of light (intensity, phase, spectrum, polarisation, etc.) propagating along these natural threads. By collecting and analysing the transmitted light, the presence of the modifying agent can be detected using a very simple transmission setup. In this configuration, the optical fibre is both used as the light conveyor and the sensing element along its whole length. Therefore, the benefits of optical fibre sensing is entirely exploited since the guided light keeps fully confined in the fibre and the effect is accumulated along the fibre, thereby upscaling its sensitivity.

\section{Pristine Spider silk, a chemically-sensitive optical fibre}
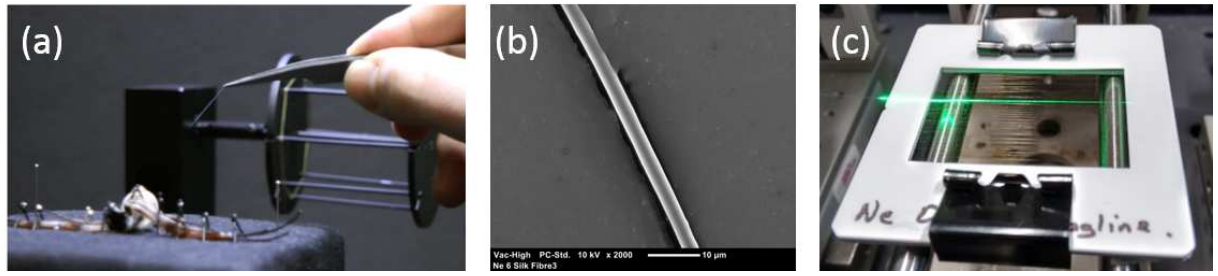

Fig. 2: (a) Dragline silk directly extracted from a female spider performed at the Oxford Silk Group. (b) SEM image of the collected silk sample. (c) Native silk placed on a holder and used as an optical fibre.

Blessed with a unique protein composition structure and incredible optical properties, spider silk thread is ideal to demonstrate this concept. Dragline silk, directly collected from the major ampullate gland of a female Nephilia edulis spider as shown in Fig. 2a, can be reeled under controlled conditions to produce uniformly spun silk fibre with a smooth surface, circular cross-section (Fig. 2b) and homogenous material properties. In its pristine condition, dragline silk transmits light as shown in Fig. 2c, with propagation losses of the order of a few $\mathrm{dB} / \mathrm{cm}$ within a measured transparency window from the visible up to $\lambda \approx 1400 \mathrm{~nm}$ and under high birefringence $\left(10^{-3}\right)$ [2].

Modifying agents with different chemical properties (for e.g. polarity) break hydrogen bonds in the silk material, which can affect the birefringence of the silk thread $[3,4]$. This change in birefringence can be easily measured using a polarimetric setup, shown in Fig. 3, to monitor the state of polarisation (SOP) of the light propagating out of the silk fibre sample, exposed to the modifying agent.

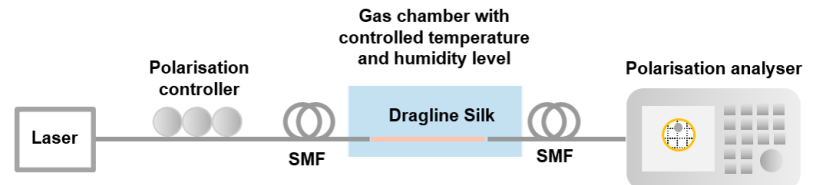

Fig. 3: Polarimetric setup used to measure polarisation transformations on a Poincar sphere of the output light when the silk thread is exposed to modyfing agents.

\section{Fibre-optic humidity sensor based on spider silk and example of application}

Exposure to humidity results in infiltration of water molecules into the silk material. They interact with the hydrophilic amino acids in the amorphous region of the proteins changing the hydrogen bonding network stabilizing the proteins resulting in a conformational change in the protein. This results in a change in the silk fibres geometry in its whole volume, which brings a substantial change in the SOP of the output signal: a rotation with a phase of $\Delta \phi$ on the Poincaré sphere could be detected as soon as water molecules were brought close to the silk thread. This process is highly reversible upon drying, as long as the humidity level does not exceed a critical threshold of $70 \%$, and can be exploited as a relative-humidity fibre-optic sensor based on spider silk with a sensitivity of $\sim 0.71 \pi \mathrm{rad} / \% \mathrm{RH}$ [5].

One appealing application for our proposed silk-based fibre optical sensor is for air and breath analysis. The peaks on Fig. 4 represent SOP phase changes caused by exhaled air detected from a subjects

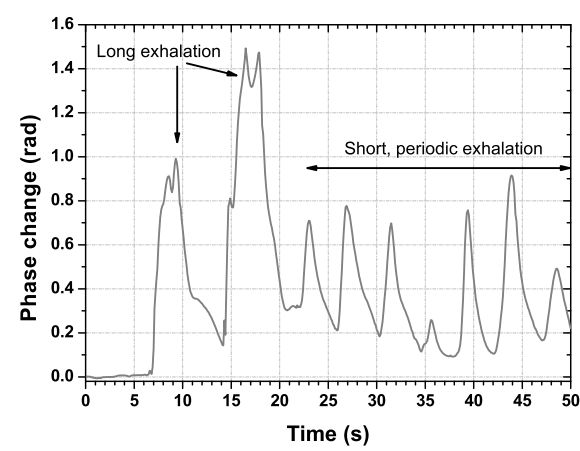

Fig. 4: Phase change of light through silk fibre due to nose-exhaled breaths (humidity level change). nose $20 \mathrm{~cm}$ away from the sensor; no air is expelled in the first 7 seconds, followed by two long exhalations and, finally, short and periodically nose-expelled air. Since human expiration is mainly composed of water vapour, we can assume that the silk sensor is essentially detecting a change in relative humidity with a very good response time. It is 
worth noting that desorption rate is lower than absorption rate in our silk sensor since the latter takes slightly more time to get back to equilibrium humidity condition. This result paves the way towards the use of silk-inspired threads for breath analysis. If the silk strands can be made exclusively selective to individual breath biomarkers, they can represent a faster, more compact, and economic alternative to the widely used laser spectroscopic detection techniques for breath analysis or to detect volatile organic compounds (VOCs) level for air quality monitoring.

\section{Discussion}

More efficient chemically-sensitive fibre-optic sensors with lower energy requirements can potentially be obtained by materials whose mechanical and optical properties change according to the surrounding environment, for e.g. spider silk threads. Advantages of silk-based fibre optic sensing are numerous: high sensitivity, fast response time, sensitive to many compounds. Having a complex molecular structure, the presence of other classes of chemical compounds in the vicinity of silk brings selective conformational reactions leading to different changes in optical properties of light guided along the silk fibre. These protein threads can, therefore, be used for sensing other target agents in trace amounts such as ammonia, ethanol, VOCs, etc. and probably represent a much better alternative for fibre-optic chemical sensing than conventional glass fibres since the latter usually require coatings, even to be used as relative humidity sensors.

Silk threads also offer a crucial degree of freedom. First of all, their material properties (degree of crystallinity, sensitivity to humidity, etc.) can be intrinsically modified during the spinning process performed at ambient conditions. Moreover, silk has the unique property to be drawn under ambient conditions (temperature, pressure), so that dyes and specific colorimetric reagents into the bulk material can potentially be incorporated in the silk bulk material, which would otherwise be denatured at the very high drawing temperatures of $\sim 2000{ }^{\circ} \mathrm{C}$ silica fibres, giving potential access to functionalised fibre sensors, exploiting the countless possibilities offered by chemistry for selective sensing.

All these qualities make these protein threads very promising for future applications. However, there are still many barriers to overcome for us to fully exploit this highly promising material for optical fibre sensing. While they are excellent fibres for proof of concept and for fundamental research, spider threads are unlikely to be accepted in their natural state by industry for upscaling of the concept into commercial devices. Hopefully, the recent progress achieved in the understanding of the silk proteins and the drawing of artificial silk [6] will allow the production of tailormade low-loss synthetic optical threads. Ideally, this would be combined with an ability to fabricate functionalised fibres for selective sensing of specific chemical compounds. This would represent the first building blocks towards the conception of a new generation of less energy-consuming, biocompatible, selective sensitive devices through a novel biomimetic bulk protein sensing approach.

\section{Acknowledgments}

This work was initiated by the European Space Agency through Ariadna study nº 14-6401 (The Silky Way) .

\section{References}

1. U. B. Kaupp, "Olfactory signalling in vertebrates and insects: differences and commonalities," Nature Reviews Neuroscience 11(3), pp. 188-200, 2010.

2. D. M. Chow, K. H. Tow, F. Vollrath, I. Dicaire, T. Gheysens, and L. Thévenaz, "Shedding light on the optical properties of spider silk fiber," in IEEE Photonics Conference, 2015.

3. K. H. Tow, D. M. Chow, F. Vollrath, I. Dicaire, T. Gheysens, and L. Thévenaz, "Spider silk: a novel optical fibre for biochemical sensing," in 24th International Conference on Optical Fibre Sensors, Proc. SPIE 9634, 2015.

4. K. H. Tow, D. M. Chow, L. Thévenaz, F. Vollrath, I. Dicaire, and T. Gheysens, "Weaving our way towards a new generation of fibre-optic chemical sensors based on spider silk," in 6th International Conference on Photonics, IEEE, 2016.

5. K. H. Tow, D. M. Chow, F. Vollrath, I. Dicaire, T. Gheysens, and L. Thévenaz, "Towards a new generation of fibre-optic chemical sensors based on spider silk threads," in 25th International Conference on Optical Fiber Sensors Conference, Proc. SPIE 10323, 2017.

6. A. Rising and J. Johansson, "Toward spinning artificial spider silk," Nature chemical biology 11(5), pp. 309$315,2015$. 